\title{
Physical properties of lensed high-redshift galaxies
}

\author{
M. Lemoine-Busserolle ${ }^{1}$, T. Contini ${ }^{2}$, R. Pello ${ }^{2}$, J.-F. Le Borgne ${ }^{2}$, \\ J.-P. Kneib ${ }^{2}$, J. Richard ${ }^{2}$, M. Kissler-Patig ${ }^{3}$, and S. F. Sanchez ${ }^{4}$ \\ ${ }^{1}$ Institute of Astronomy, Madingley Road, CB3 OHA, Cambridge - UK \\ email: lemoine@ast.cam.ac.uk \\ ${ }^{2}$ LAOMP, 14 Avenue E. Belin, F-31400 Toulouse - France \\ ${ }^{3}$ ESO, Karl-Schwarzschild-Str.2, 85748 Garching - Germany, \\ ${ }^{4}$ CAHA, C/Jesus Durban Remon 2-2, E-04004 Almeria - Spain
}

\begin{abstract}
We report the results obtained from optical and near-infrared spectroscopy and also broad-band photometry of lensed low-luminosity galaxies $(1.3 \leqslant z \leqslant 2.3)$ located in the core of lensing clusters. The amplification factor allowed us to obtain the physical properties (SFR, abundance ratios, mass, age of the burst, dust contents, etc.) of star-forming galaxies, 1 to 2 magnitudes fainter than galaxies in a blank field, using the same indicators as in studies of low-redshift galaxies. A part of this study was concentrated on the dynamics of distant lensed galaxies using Integral Field Spectroscopy (SINFONI/VLT science verification programme).
\end{abstract}

\section{Introduction}

Clusters of galaxies acting as Gravitational Telescopes constitute a unique tool to study high- $z$ galaxies. Indeed, the large magnification factors of galaxies that are close to the critical lines (typically 1 to 3 magnitudes) can be used to probe the physical properties of intrinsically faint high-redshift galaxies, which would otherwise be beyond the limits of conventional spectroscopy (see e.g. Pelló et al. 2003 for a review). The main stellar/interstellar features observed in spectra of star-forming galaxies from the UV to the optical can be use as fundamental tools to determine the properties of the stellar populations and of the ionised gas from integrated spectra. Although scarce, studies of the UV rest-frame spectra of high-redshift galaxies have begun to uncover interesting information on their stellar populations, star formation histories, and chemical abundances (cf. Pettini et al. 2000; de Mello et al. 2000; Mehlert et al. 2002; Erb et al. 2003; Rix et al. 2003). Such detailed spectroscopic analysis of individual objects are complementary to the relative crude broad-band photometric studies of the stellar populations of Lyman-break Galaxies (hereafter LBGs) such as carried out by Papovich et al. (2001) and Shapley et al. (2003). In addition, now spectroscopy with near-infrared spectrographs on $8-10 \mathrm{~m}$ telescopes give access to the most relevant rest-frame optical emission-line diagnostics (Balmer lines, [O II] $\lambda \lambda 3726,3728$ [O III] $\lambda \lambda 4959,5007$ [N II] $\lambda 6584$, etc.) which allow the study of the rest-frame optical properties of galaxies at $1.5 \leqslant z \leqslant 4$. Covering simultaneously the rest-frame UV-to-optical spectra of such high- $z$ galaxies is of great interest, e.g. to compare extinction and abundance determinations obtained in these two windows. 


\section{Observations and analysis}

The main goal of these studies is to derive the physical properties of distant galaxies using the same parameter space as for galaxies in the local Universe. We report, in this contribution, the results obtained on 4 lensed low-luminosity distant galaxies $(1.3 \leqslant$ $z \leqslant 2.3$ ) located in the core of the lensing clusters AC114, CL2244-02, and A370. The amplification factor allowed us to obtain, for the first time, emission-line measurements on star-forming galaxies as faint as $M_{\mathrm{B}} \sim-20$, thus 1 to 2 magnitudes fainter than in previous studies of LBGs at $z \sim 3$.

In this study, we used three sets of data. Two different spectroscopic datasets, namely the rest-frame UV and optical spectra obtained with the optical spectrograph FORS2 and the near-infrared spectrograph ISAAC respectively on the VLT, and BVRIJK photometry deduced from available public images. From these combined multi-wavelength observations using at the same time spectroscopic data and broad-band photometry, the main applications have been the determination of : (a) Star formation rate: deduced from the optical emission-line fluxes of $\mathrm{H} \alpha$ and also from the intensity of the continuum of the UV spectra. (b) Chemical abundances $\&$ metallicity : the optical emission-line fluxes of [O III] $\lambda \lambda 4959,5007$ [O III] $\lambda \lambda 4959,5007 \mathrm{H} \beta$ and [N II] $\lambda 6584$ were used to estimate the oxygen-to-hydrogen $(\mathrm{O} / \mathrm{H})$ (i.e. the metallicity) and the nitrogen-to-oxygen $(\mathrm{N} / \mathrm{O})$ abundance ratios in the gas. The UV continuum slope gives an estimation of the $\mathrm{O} / \mathrm{H}$ abundance ratio from the stellar population. (c) Age of the burst: deduced from a comparison of the shape of the stellar lines of the UV rest-frame spectrum with predictions of evolutionary synthesis models (such as STARBUST99). (d) Reddening : deduced from the multicolour Spectral Energy Distribution fitting by the predictions of evolutionary synthesis models. The comparison between the star formation rate deduced from the UV continuum and the $\mathrm{H} \alpha$ optical emission line also give an estimation of the internal extinction. (e) Kinematics (including the determination of dynamical/virial masses) : deduced from the strong optical emission lines' velocity widths and rotational velocities traced by the ionised gas. This complete set of properties deduced for the lensed sample increase the existing samples of bright high redshift galaxies, both in luminosity and in redshift. This allowed, for the first time, a comparative study of the physical properties of galaxies from the early Universe with those of nearby samples (see Fig. 1a). For example, the behaviour of $A C 114-S 2$ and $A C 114-A 2$ in terms of metallicity is very different, and they are also different from typical LBGs at $z \sim 3$. AC114-S2 is a low-metallicity object $\left(\mathrm{Z} \sim 0.03 \mathrm{Z}_{\odot}\right)$ with a low $\mathrm{N} / \mathrm{O}$ ratio, similar to those derived in the most metal-poor nearby Hit galaxies. On the contrary, $A C 114-A 2$ is a metal-rich galaxy $\left(\mathrm{Z} \sim 1.5 \mathrm{Z}_{\odot}\right)$ with a high $\mathrm{N} / \mathrm{O}$ abundance ratio, similar to those derived in the most metal-rich massive SBNGs. The position of $A-C L 2244-02$ and $M S 1512-c B 58$, a lensed luminous LBG (Teplitz et al. 2000), is intermediate between these two extremes showing abundance ratios typical of low-mass SBNGs and intermediate-redshift galaxies.

Thanks to the tangential stretching induced by gravitational lensing, line profiles can be spatially resolved. We have designed a part of this research programme to carry out a near-infrared spectroscopic follow up of spatially resolved lensed galaxies. In the case of the science verification programme of SINFONI/VLT), we obtained 2D velocity maps from the $\mathrm{H} \alpha$ emission line of a star-forming lensed galaxy $(z=1.341)$, behind the core of the lens cluster Abell 370. Contrary to classical long-slit techniques (Lemoine-Busserolle et al. 2003), the use of 3D spectroscopy with a well suited spatial sampling allowed for a better correction for geometrical projection effects, the measure of $2 \mathrm{D}$ fluctuations in velocity dispersion across the objects, the detection and study of velocity fields, and improvement in the $\mathrm{S} / \mathrm{N}$ ratio (see Fig. 1b). 

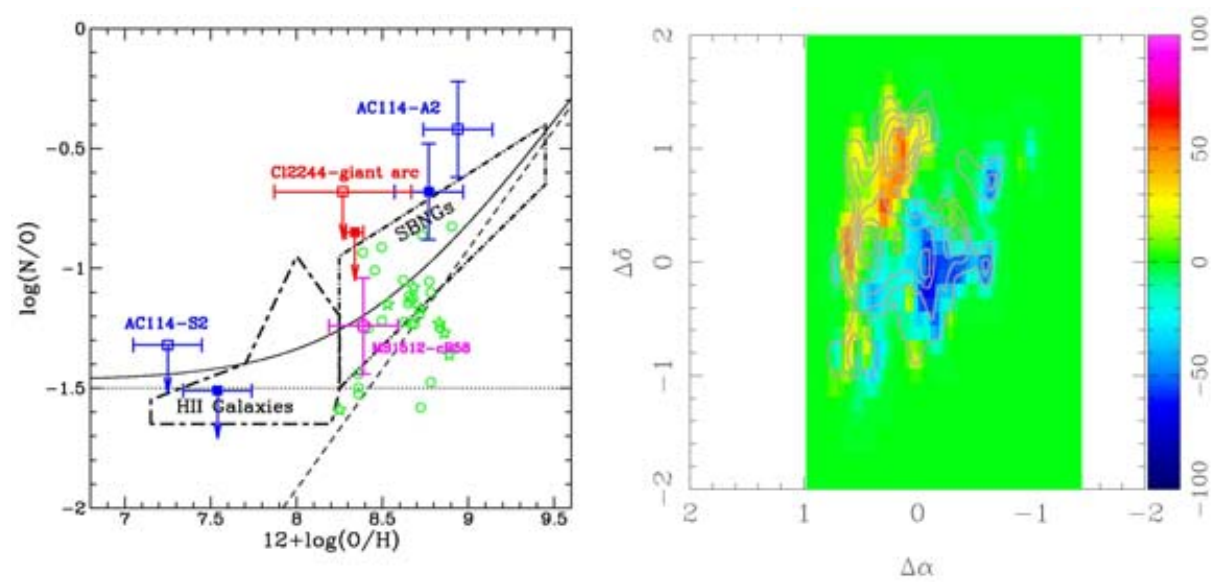

Figure 1. (a): N/O versus $\mathrm{O} / \mathrm{H}$ relation for nearby (HII galaxies and SBNGs) and high- $z$ star-forming galaxies. The location of the lensed galaxies is shown without extinction correction (empty squares) and assuming $\mathrm{E}(\mathrm{B}-\mathrm{V})=0.3$ (filled squares). (b): the $\mathrm{H} \alpha$ velocity map in $\mathrm{km} / \mathrm{s}$ obtained by fitting the emission line of each spectrum to a single Gaussian function, with the $\mathrm{H} \alpha$ intensity overlaid as contours, after correction for lensing effects.

\section{Results and conclusions}

The aim of this study was to determine the physical properties, i.e. the star formation rate, reddening, chemicals abundances, mass, and age of the stellar population from the UV (stellar population) and optical (ionised gas) rest-frame, using the same indicators in study of low-redshift galaxies as in a sample of lensed high-redshift galaxies. We found that the source of the giant arc $A-C L 2244-02(z=2.24)$ seems to be a starburst galaxy showing a face-on disk with a sub-Solar metallicity and which has experienced a burst with an age of between 10 and 15 Myr. In the case of $A C 114-A 2(z=1.867)$, an evolved starburst with a high metallicity $\left(Z \sim 1.3 \mathrm{Z}_{\odot}\right)$, very massive, and with a large amount of dust was found; while $A C 114-S 2(z=1.869)$ was found to be a young starforming HII galaxy with a low mass and a sub-Solar metallicity. In the particular case of $A 370-A 5$ ( $z=1.341)$, we obtained a velocity gradient which traces a rotation curve of $\sim 110 \mathrm{~km} / \mathrm{s}$ in a disk-like geometry. The lensed galaxies of this sample exhibit different physical properties in terms of abundance ratios, mass, SFRs, M/L, and reddening. These preliminary results from this small sample suggest different star formation histories for distant galaxies of different luminosities.

\section{Acknowledgements}

Part of this work was supported by the French Conseil Régional de la Martinique, by the French Centre National de la Recherche Scientifique and by the Marie Curie Research Training Network Euro3D; contract No. HPRN-CT-2002-00305.

\section{References}

Erb, D. K., Shapley, A. E., Steidel, C. C., Pettini, et al., 2003, ApJ, 591, 101

de Mello, D. F., Leitherer, C., Heckman, T. M., 2000, ApJ, 530, 251

Lemoine-Busserolle, M., Contini, T., Pelló, et al., 2003, A\&A, 397, 839

Pello, R., Richard, J., Schaerer, D., Le Borgne, J. F., Kneib, J. P., 2003, SF2A-2003, 33

Pettini, M., Steidel, C. C., Adelberger, K. L., et al., 2000, ApJ, 528, 96

Shapley, A. E., Steidel, C. C., Pettini, M., Adelberger, K. L., 2003, ApJ, 588, 65

Teplitz, H. I., McLean, I. S., Becklin, E. E., et al., 2000, ApJ, 533, L65 